\title{
Trends in and perspectives on extracorporeal membrane oxygenation for severe adult respiratory failure
}

\author{
Tomohito Sadahiro, MD, PhD $\cdot$ Shigeto Oda, MD, PhD \\ Masataka Nakamura, MD · Yo Hirayama, MD, PhD \\ Eizo Watanabe, MD, PhD \\ Yoshihisa Tateishi, MD, PhD \\ Koichiro Shinozaki, MD, PhD
}

Received: 20 January 2011

(C) The Japanese Association for Thoracic Surgery 2012

\begin{abstract}
Various approaches such as ventilator management involving lung-protective ventilation, corticosteroids, prone positioning, and nitric oxide have failed to maintain sufficient lung oxygenation or appropriate ventilation competence in very severe acute respiratory distress syndrome (ARDS). Extracorporeal membrane oxygenation (ECMO) has been aggressively introduced for such patients, although in only a few institutions. The clinical usefulness of ECMO in a large-scale multicenter study (CESAR trial, 2009) and continued development/improvement of ECMO devices have facilitated performance of ECMO, with further increase in the number of institutions adopting ECMO therapy. Clinical usefulness of ECMO was documented in many cases of severe ARDS secondary to influenza A (H1N1) 2009 infection. ECMO requires establishment of an appropriate management system to minimize fatal complications (e.g., hemorrhage), which requires a multidisciplinary team. This, in combination with a new technique, interventional lung assist, will further extend the indications for ECMO. ECMO can be expected to gain importance as a respiratory support technique.
\end{abstract}

Key words Acute respiratory distress syndrome $(\mathrm{ARDS}) \cdot$ Extracorporeal life support (ECLS) ·

This review was submitted at the invitation of the editorial committee.

T. Sadahiro $(\bowtie) \cdot$ S. Oda $\cdot$ M. Nakamura $\cdot$ Y. Hirayama E. Watanabe $\cdot$ Y. Tateishi $\cdot$ K. Shinozaki

Department of Emergency and Critical Care Medicine, Chiba University Graduate School of Medicine, 1-8-1 Inohana, Chuoku, Chiba 260-8677, Japan

Tel. +81-43-226-2372; Fax +81-43-226-2371

e-mail: sadahiro-t@faculty.chiba-u.jp
Hemorrhagic complication - Interventional lung assist (ILA) $\cdot$ Lung protective ventilation strategy

\section{Introduction}

Despite recent advances in the field of critical care medicine, severe acute respiratory failure remains a critical condition refractory to treatment and associated with poor survival. Acute respiratory distress syndrome (ARDS) is a representative type of severe acute respiratory failure. The first proposal for internationally coordinated diagnostic criteria for ARDS at the American-European Consensus Conference on ARDS in $1994^{1}$ resulted in rapid advances in elucidation of the pathophysiology of ARDS as well as extensive investigation of ventilator management strategies and various therapeutic approaches involving corticosteroids, prone positioning, and nitric oxide. The importance of lungprotective ventilation strategy in ventilator management has recently been emphasized, and various combinations of multiple lung protective ventilation strategies, including low-tidal volume ventilation as the key component, have been attempted in the treatment of ARDS. ${ }^{2}$

Unfortunately, these therapeutic approaches have failed to maintain sufficient lung oxygenation or appropriate ventilation competence in a small number of patients with very severe ARDS. Extracorporeal membrane oxygenation (ECMO) has been aggressively introduced to the treatment of such patients, who are most refractory to or not salvageable with conventional therapeutic interventions in a small number of medical institutions. Because of the limited number of patients with such severe respiratory failure, the clinical usefulness of ECMO had mostly been considered only in case reports 
and single-center cohort studies with a relatively low level of evidence. In 2009, however, a multicenter randomized controlled trial (RCT) investigating the clinical usefulness of ECMO in adults with severe respiratory failure, the Conventional Ventilatory Support versus Extracorporeal Membrane Oxygenation for Severe Adult Respiratory Failure (CESAR) trial, demonstrated a significant increase in the percentage of patients surviving up to 6 months without disability among those undergoing ECMO compared with those receiving conventional management. ${ }^{3}$ These results thus settled a long controversy regarding the clinical usefulness of ECMO in adults. More recently, the clinical usefulness of ECMO was documented in many cases of severe ARDS secondary to the influenza A (H1N1) 2009 infection. ${ }^{46}$ In addition to infectious diseases, ECMO has been applied to the treatment of burn injury with $\mathrm{ARDS}^{7}$ and traumatic lung injury, ${ }^{8}$ as a bridge to lung transplantation, ${ }^{9}$ and for management after lung transplantation, ${ }^{10}$ with excellent therapeutic effects. The development and improvement of medical devices for ARDS are continuing in parallel: An extracorporeal circulation system with a long-term durable antithrombogenic surface coating ${ }^{11}$ and a new double-lumen catheter realizing a single-venous cannulation strategy in adults ${ }^{12}$ are both close to practical use. The present review discusses in detail the current situation and future of ECMO as a treatment option for severe respiratory failure.

\section{Definitions}

Some confusion exists regarding the terms for designating mechanical circulatory support modalities applied to the treatment of severe respiratory failure. This confusion is mainly ascribable to regional differences. In Japan and some other Asian countries, mechanical circulatory support for patients with circulatory failure is conventionally designated percutaneous cardiopulmonary support (PCPS), whereas the term ECMO is used exclusively to designate mechanical circulatory support for patients with respiratory failure without circulatory failure. More globally, however, the term ECMO is used for mechanical support modalities regardless of their main aim - respiratory or circulatory support - and the term PCPS is scarcely used outside Asia. In addition to ECMO and PCPS, mechanical support systems designed mainly for carbon dioxide removal in patients with hypercapnia are designated extracorporeal carbon dioxide removal (ECCO2R) or interventional lung assist (ILA) systems (see below). Extracorporeal gas exchange techniques, including ECMO, ECCO2R, and ILA, are collectively termed techniques of extracorporeal lung
Table 1 Numbers of papers in PubMed

\begin{tabular}{lcc}
\hline Terms & \multicolumn{2}{l}{ Results (no.) } \\
\cline { 2 - 3 } & All & Last 1 year \\
\hline Extracorporeal membrane & 4913 & 450 \\
$\quad$ oxygenation (ECMO) & & \\
Extracorporeal life support (ECLS) & 1059 & 113 \\
Percutaneous cardiopulmonary & 586 & 34 \\
$\quad$ support (PCPS) & 268 & 28 \\
Extracorporeal lung assist (ECLA) & 213 & 11 \\
Extracorporeal carbon dioxide & & \\
$\quad$ removal (ECCO $\mathrm{R}$ ) & 68 & 15 \\
Interventional lung assist (ILA) & \multicolumn{2}{c}{15} \\
\hline
\end{tabular}

assist (ECLA) or extracorporeal life support (ECLS). The term ECLS, in particular, is used in a case registry maintained as a major function of the Extracorporeal Life Support Organization (ELSO), an international consortium of health care professionals and scientists established in 1989 to promote broad multidisciplinary collaboration in the development and evaluation of novel treatments to support failing organ systems. The multiple terms currently used in parallel may therefore be unified as ECLS in the future. Nevertheless, the terms ECLS and ECMO are currently used nearly as synonyms in some reports, ${ }^{13}$ indicating that they are not yet clearly understood as distinct terms. Table 1 summarizes the results of a keyword search of PubMed using the abovementioned terms as key words. Search results with and without limitation of publication date (within 1 year of the search date, December 21, 2010) are presented for comparison. Results indicate a current tendency for ECMO to be used most frequently among all terms examined and in preference to ELSO.

\section{ECMO modalities and system}

ECMO is performed in two modes: venovenous $(\mathrm{V}-\mathrm{V})$ mode, which is exclusively used for respiratory support in severe respiratory failure, and venoarterial (V-A) mode, which is capable of providing both respiratory and circulatory support (Fig. 1). Table 2 summarizes the advantages and disadvantages of these two modes. In either ECMO mode, blood is withdrawn from a catheter inserted into a blood vessel with a centrifugal pump and then returned to the body via another catheter after extraocorporeal gas exchange using an artificial lung. The procedures for ECMO are described in detail in a book published by ELSO ${ }^{14}$ Online guidelines also are available from the ELSO website (http://www.elso.med. umich.edu/Guidelines.html) and from "Interventional Procedure Guidance 39, ECMO in Adults" published by 
Fig. 1 Schema for venovenous $(V-V)$ and venoarterial $(V-A)$ extracorporeal membrane oxygenation $(E C M O)$

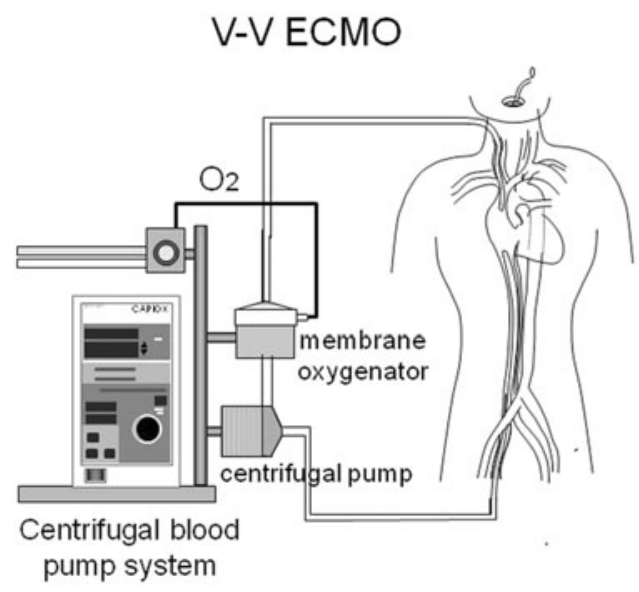

Cavo-jugular bypass

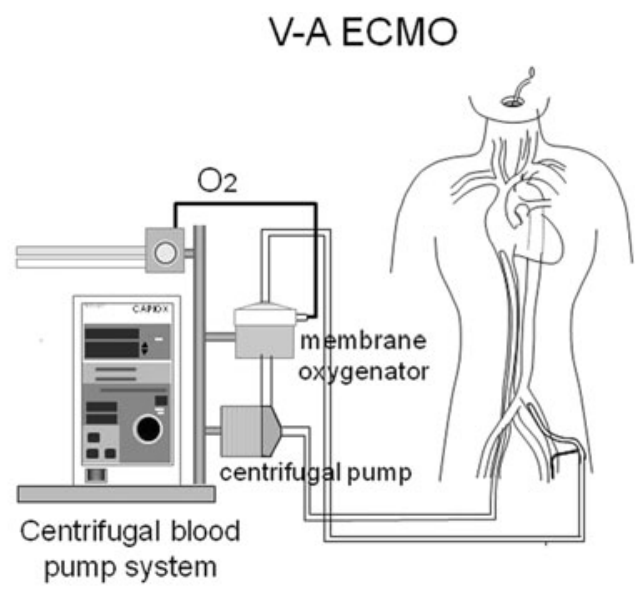

Right atrio-femoral bypass
Table 2 Comparison of venovenous and venoarterial ECMO

\begin{tabular}{|c|c|}
\hline V-V ECMO & V-A ECMO \\
\hline $\begin{array}{l}\text { Uniformly improves } \\
\text { oxygen pressure. Ensures } \\
\text { delivery of oxygenated } \\
\text { blood to the brain }\end{array}$ & $\begin{array}{l}\text { Oxygenated blood may not be } \\
\text { delivered to the brain at high } \\
\text { natural cardiac output }\end{array}$ \\
\hline $\begin{array}{l}\text { Lower incidence of } \\
\text { complications, such as } \\
\text { blood flow obstruction } \\
\text { (e.g., lower extremity } \\
\text { ischemia) and embolism }\end{array}$ & $\begin{array}{l}\text { Usually involves lower extremity } \\
\text { bypass to maintain blood flow } \\
\text { in the lower extremity }\end{array}$ \\
\hline $\begin{array}{l}\text { Involves no arterial } \\
\text { puncture. Easy } \\
\text { hemostasis on weaning }\end{array}$ & $\begin{array}{l}\text { Generally requires suture of the } \\
\text { arterial puncture site on } \\
\text { weaning }\end{array}$ \\
\hline $\begin{array}{l}\text { Inefficient oxygenation due } \\
\text { to blood recirculation in } \\
\text { the circuit }\end{array}$ & $\begin{array}{l}\text { Oxygenation proportional to } \\
\text { blood flow rate can be expected } \\
\text { without blood recirculation in } \\
\text { the circuit }\end{array}$ \\
\hline $\begin{array}{l}\text { No circulatory support } \\
\text { expected }\end{array}$ & Capable of circulatory support \\
\hline
\end{tabular}

the National Institute for Health and Clinical Excellence in 2004 (http://www.nice.org.uk/nicemedia/live/11032/ 30652/30652.pdf). Tables 3 and 4 summarize details of current entry criteria for and relative contraindications to ECMO, respectively. The current characteristics of the three essential components of a V-V ECMO system for adults, catheters, pumps, and artificial lungs (extracorporeal membrane oxygenators) are briefly described below.

\section{Catheters}

The oxygen delivery capability in patients undergoing ECMO is determined by various parameters including hemoglobin level and oxygen saturation. Of these parameters, the blood flow rate is most easily and flexibly adjusted during ECMO. For obtaining a sufficient
Table 3 Indications for ECMO for adult respiratory failure

1. For hypoxic respiratory failure due to any cause (primary or secondary), ECLS should be considered when the risk of mortality is $\geq 50 \%$ and is indicated when the risk is $\geq 80 \%$. a. A $50 \%$ mortality risk can be identified by a $\mathrm{PaO}_{2} / \mathrm{FiO}_{2}$ $<150 \mathrm{mmHg}$ on $\mathrm{FiO}_{2}>90 \%$ and/or a Murray score of 2-3.

b. An $80 \%$ mortality risk can be identified by a $\mathrm{PaO}_{2} / \mathrm{FiO}_{2}$ $<80 \mathrm{mmHg}$ on $\mathrm{FiO}_{2}>90 \%$ and a Murray score of 3-4.

2. $\mathrm{CO}_{2}$ retention due to asthma or permissive hypercapnia with a $\mathrm{PaCO}_{2}>80 \mathrm{mmHg}$ or inability to achieve safe inflation pressures (Pplat $\leq 30 \mathrm{cmH}_{2} \mathrm{O}$ ) is an indication for ECLS.

3. Severe air leak syndromes.

Pplat, plateau pressure

From Patient specific supplements to the ELSO general guidelines. Available at: http://www.elso.med.umich.edu/Guidelines.html

Table 4 Contraindications to ECMO

There are no absolute contraindications to ECLS because each patient is considered individually with respect to risks and benefits. There are conditions, however, that are known to be associated with a poor outcome despite ECLS and can therefore be considered relative contraindications.

1. Mechanical ventilation at high settings $\left(\mathrm{FiO}_{2}>0.9\right.$, Pplat $>30$ ) for $\geq 7$ days

2. Major pharmacological immunosuppression (absolute neutrophil count $<400 / \mathrm{mm}^{3}$ )

3. Central nervous system hemorrhage that is recent or expanding

Patient Specific Supplements to the ELSO General Guidelines (http://www.elso.med.umich.edu/Guidelines.html)

blood flow rate, the selection of catheters used for ECMO is crucial. In particular, optimization of catheter size and length is the key to successful ECMO. However, few options are currently available in Japan with respect to the size and length of drainage/reinfusion catheters appropriate for ECMO in V-V mode because ECMO has been performed mainly in the $\mathrm{V}$-A mode in Japanese intensive care unites (ICUs). We therefore perform ECMO in V-V mode with a catheter for drainage inserted 
from the femoral vein into the inferior vena cava and another catheter for reinfusion inserted from the right internal jugular vein and placed near the inlet of the right atrium. The V-V ECMO was previously generally performed with a catheter for drainage inserted from the right internal jugular vein and another for reinfusion inserted from the femoral vein (atriofemoral flow). Rich et al., however, reported that flow reversal (femoroatrial flow) by exchanging the insertion sites for the two catheters provided higher maximum extracorporeal flow and pulmonary arterial mixed venous oxygen saturation, and it required comparatively less flow to maintain an equivalent mixed venous oxygen saturation than did conventional atriofemoral flow. ${ }^{15}$ The femoroatrial flow method proposed by Rich et al. has since been the global mainstream for V-V ECMO.

Whereas ECMO in children with a body weight of $\leq 15 \mathrm{~kg}$ uses a single $18 \mathrm{~F}$ double-lumen catheter (with the advantage of a minimum number of puncture sites), ECMO in adults uses two single-lumen catheters inserted at two sites (dual cannulation) as noted above to ensure sufficient blood flow. Under these circumstances, recent clinical application of a bicaval dual-lumen catheter developed by Avalon Laboratories (Rancho Dominguez, CA) in the United States and Europe has enabled high-flow ECMO with a single-cannulation strategy. ${ }^{12}$ There is a possibility that single-venous cannulation not only reduces the risk of complications associated with catheter insertion (which most frequently occur on insertion from the femoral vein) but also facilitates postural change and mobilization of the patient during ECMO, thereby potentially improving the safety of management of conscious patients. Further studies are needed to evaluate the usefulness and safety of this new catheter.

\section{Pumps}

Many medical institutions currently prefer a centrifugal blood pump to a roller occlusion blood pump for ECMO in adults. Although a centrifugal pump is generally believed to be associated with a lower risk of complications during ECMO (e.g., damage to extracorporeal blood circuit tubing and hemolysis) than a roller occlusion pump, ${ }^{16}$ the incidence of complications associated with use of centrifugal pumps for ECMO reportedly varies depending on such factors as the shape of the impeller. ${ }^{17,18}$ In addition, a roller pump has been reported to be less hemolytic than a centrifugal pump during blood circuit occlusion. ${ }^{19}$ Thus, blood pumps for ECMO should be carefully evaluated and selected. At present, almost all commercial blood pumps use a pump head and a blood circuit with heparin-coated surfaces, which in combination with an artificial lung (extracorporeal membrane oxygenator) with improved biocompatibility allows prolonged ECMO. ${ }^{11}$

\section{Membrane oxygenators}

Extracorporeal membrane oxygenators containing hollow-fiber membranes were introduced to clinical practice during the 1980s. More recently, coating of the membrane surface with silicone and heparin has greatly prolonged the lifetime of membrane oxygenators. In Japan, devices allowing easy priming/purging of the blood circuit in emergency situations tend to be preferred. Although a new membrane oxygenator recently developed in Japan demonstrated excellent long-term durability allowing continuous use for 3 months or even longer without heparin administration in an animal study, ${ }^{20}$ membrane change at intervals of approximately 1 week must be considered in actual clinical practice when currently available commercial products are used. Further improvement of the long-term biocompatiblity (e.g., antithrombogenicity) of membrane oxgenators is required for wider dissemination of ECMO.

\section{Historical context of early studies on ECMO}

The history of ECMO began during the 1970s. During ventilator management of severe ARDS with advanced hypoxemia and hypercapnia, it is sometimes difficult to maintain arterial blood oxygen saturation or to adjust blood $\mathrm{pH}$ within an appropriate range by reducing the blood $\mathrm{CO}_{2}$ level simply by changing the operational conditions of the ventilator used to maximize its performance. In such cases, there is little hope for survival of the patient with further continuation of ventilator management. In 1972, Hill et al. reported the first case of survival achieved by ECMO in a patient with acute posttraumatic respiratory failure. ${ }^{21}$ In 1974 , a multicenter RCT attempting to demonstrate the clinical efficacy of ECMO during severe acute respiratory failure was begun at nine medical institutions in the United States. The results of this study that had enrolling 90 adult patients and was published in 1979 by Zapol et al. ${ }^{22}$ failed to demonstrate a significant difference in survival rate between patients undergoing ECMO versus those with conventional ventilation $(9.5 \%$ vs. $8.3 \%)$. This lack of difference may have been due to performance of the study in the early days of the field using techniques that are not standard today: ${ }^{23}$ (1) The procedures for ventilator management employed were markedly different from those used at present; (2) ECMO in all study subjects was performed using $\mathrm{V}$-A access and not $\mathrm{V}-\mathrm{V}$ access, the present standard of treatment; (3) the level of anticoagu- 
lation used was much higher than current standards due to the limited antithrombogenicity of ECMO devices used at that time, leading to a marked increase in blood transfusion volume in patients undergoing ECMO compared with those with conventional ventilation; and (4) the mean duration of mechanical ventilation prior to ECMO was 9.6 days (i.e., ECMO was initiated after ventilator-associated lung injury had already been induced). The results of this trial were considered evidence that ECMO is not clinically useful in adults. Hence, use of ECMO for severe respiratory failure in adults subsequently attracted little interest.

For neonatal and pediatric critical care, in contrast, the clinical usefulness of ECMO for treating severe respiratory failure was documented in multiple studies published during the 1980s and 1990s. ${ }^{24-27}$ In 1996, results of an RCT by the U.K. collaborative ECMO trial group yielded clear evidence of the usefulness of ECMO in neonates. ${ }^{28}$ ECMO treatment in neonates and children has since attained a firm position as "the last card to play" in the treatment of severe respiratory failure. ${ }^{13}$

\section{Changes in ventilator management in ARDS and mechanism of effectiveness of ECMO}

The greatest advance made in the treatment of ARDS following the early attempts to introduce ECMO into clinical practice has been improvement of ventilator management strategies. ${ }^{29}$ It is now widely recognized that repeating ventilation at a high airway pressure with a mechanical ventilator is harmful and that respiratory management that minimizes the resulting ventilatorinduced lung injury (VILI) - including barotrauma, volotrauma, atelectrauma, and biotrauma-improves survival. ${ }^{30}$ In addition to a low-tidal volume strategy involving a reduction of tidal volume from the conventional $10 \mathrm{ml} / \mathrm{kg}$ to approximately $6 \mathrm{ml} / \mathrm{kg}$, respiratory management adopting the concept of lung protective strategy (e.g., permissive hypercapnia and open-lung strategy) has been recommended. ${ }^{2}$ Clinical application of new ventilation modes such as airway pressure release ventilation (APRV) and high-frequency oscillatory ventilation $^{31}$ has been attempted in parallel.

Another noteworthy event in the history of treatment of ARDS is the establishment of a simple and clear definition of ARDS in 1994, ${ }^{1}$ which stimulated active and extensive clinical studies and led to rapid progress in elucidation of the pathophyisology of ARDS as well as establishing novel approaches to the treatment of it. Using this definition, the clinical usefulness of therapeutic approaches involving corticosteroids, prone positioning, and nitric oxide has been investigated in several large-scale RCTs. ${ }^{32-34}$ Results indicated that these approaches should be regarded as therapeutic options to be considered in life-threatening conditions, rather than routinely applied to all patients with ARDS. However, inclusion of the lung-protective ventilation strategy noted above in setting operating conditions for a ventilator during management of patients with severe ARDS makes it more difficult to maintain arterial blood oxygen saturation or adjust blood $\mathrm{pH}$ according to the concept of permissive hypercapnia by reducing the blood $\mathrm{CO}_{2}$ level. Initiation of ECMO in patients without successful control of $\mathrm{SpO}_{2}$ and blood $\mathrm{pH}$ under "lung-protective" operating conditions of the ventilator is a reasonable therapeutic approach because the membrane oxygenator, an artificial lung, in the ECMO system compensates for the impaired function of the patient's biological lungs and achieves efficient oxygenation and $\mathrm{CO}_{2}$ elimination even under "lung-protective" mechanical ventilation. In addition, concomitant use of a membrane oxygenator allows operation of a mechanical ventilator at a lower oxygen concentration setting, thereby reducing the risk of oxygen toxicity.

ECMO per se is an invasive procedure and is associated with the risk of various complications such as hemorrhage. Nevertheless, a natural trend toward aggressive application of it to cases in which benefits outweigh risks yielded a series of publications reporting successful results during the last half of the 1990s and thereafter. In a prospective epidemiological study published in 1997 from Germany, Lewandowski et al. investigated the usefulness of a clinical algorithm for management of ARDS involving ECMO as "the last resort." 35 Briefly, 122 patients with severe ARDS referred to a university hospital underwent advanced treatment involving a lungprotective ventilation strategy. Those not responding to advanced treatment further underwent ECMO. The survival rates they reported were excellent: for the no-ECMO group (patients with ARDS undergoing advanced treatment but not ECMO) $89 \%$; ECMO group 55\%; and overall $75 \%$. These percentages far exceed those reported earlier. Bartlett et al. had reported a survival rate of $39 \%$ in a prospective study of acute hypoxic respiratory failure published in $1986 .{ }^{36}$ Vasilyev et al. reported a comparable survival rate of $45 \%$ in an international, multicenter, prospective survey of acute respiratory failure published in $1995 .{ }^{37}$ Consistent with the results of Lewandowski et al., successful results in patients with ARDS achieved with clinical algorithms involving ECMO with slight variations were reported thereafter, although most were published in the form of singlecenter retrospective case series ${ }^{38-43}$ (Table 5).

While the first RCT of ECMO, conducted during the second half of the $1970 \mathrm{~s}^{22}$ failed to demonstrate its clini- 
Table 5 Randomized control trials and cohort studies of ECMO for ARDS

\begin{tabular}{llllr}
\hline Author & $\begin{array}{l}\text { Year of } \\
\text { publication }\end{array}$ & $\begin{array}{l}\text { Cases } \\
\text { (no.) }\end{array}$ & $\begin{array}{l}\text { Hospital } \\
\text { Survival (\%) }\end{array}$ & Ref. \\
\hline RCTs & & & & \\
$\quad$ Zapol & 1979 & 48 & 9.5 & 22 \\
$\quad$ Peek (CESAR) & 2009 & 90 & 63 & 3 \\
Cohort studies & & & & \\
Lewandowski & 1997 & 49 & 55 & 35 \\
Peek & 1997 & 50 & 60 & 41 \\
Ullrich & 1999 & 13 & 62 & 38 \\
Mols & 2000 & 17 & 55 & 39 \\
Lindén & 2000 & 17 & 76 & 42 \\
Hemmila & 2004 & 255 & 53 & 2 \\
Beiderlinden & 2006 & 32 & 53 & 40 \\
Nehra & 2008 & 81 & 53 & 43 \\
\hline RCT, randomized & & & & \\
\hline
\end{tabular}

$\mathrm{RCT}$, randomized controlled trial

cal efficacy, a series of reports supporting its clinical usefulness were published during the second half of the 1990s (Table 5), highlighting its potential as a promising treatment option for severe ARDS. Nevertheless, it should be noted that most of these reports were singlecenter cohort studies conducted at a limited number of medical institutions with substantial experience in ECMO, a treatment extremely difficult to perform. As a consequence, a number of experts in the field were skeptical of these reports despite the excellent survival rates achieved.

Although the highest level of evidence supporting the clinical usefulness of ECMO can only be provided by a multicenter RCT, simple application of an ordinary design for an RCT to investigate the clinical efficacy of ECMO appeared difficult for the following reasons. First, ECMO is a special technique that requires much experience and expertise, and it is not routinely employed in every medical institution. As a consequence, the study design for a controlled trial of ECMO inevitably involves the transfer of study subjects with severe ARDS to particular medical institutions capable of performing ECMO at certain time points according to clearly defined criteria. However, it is usually difficult to design the unified protocol required for this owing to differences in local methods of treatment. In addition, a study design in which subjects undergoing and not undergoing ECMO are treated at different medical institutions raises the legitimate question of whether it is appropriate simply to compare therapeutic results in subjects with and without ECMO obtained at different medical institutions. Despite all these difficulties, which have hindered establishment of the highest level of evidence for clinical usefulness of ECMO, an ambitious RCT, the Conventional Ventilatory Support versus Extracorporeal Membrane Oxygenation for Severe Adult Respiratory Failure
(CESAR) trial, ${ }^{3}$ was conducted in the United Kingdom between 2001 and 2006.

\section{CESAR trial}

The CESAR trial was conducted in 180 adults with reversible severe ARDS at 104 centers in the United Kingdom between July 2001 and June 2006. The study design for this trial had two notable features: (1) it covered the entire United Kingdom and (2) patients randomly assigned to ECMO or conventional ventilation were treated at different medical institutions. Patients assigned to ECMO were transferred exclusively to the Glenfield Hospital, Leicester, which had 17 years of experience and was the only ELSO-recognized adult ECMO center in the United Kingdom at trial initiation. Those assigned to conventional management were treated in conventional treatment centers (CTCs), either those acknowledged by Critical Care Network leads (where established) to provide appropriately high standards of conventional care for ECMO-eligible patients, or units that treated $\geq 350$ patients per year and could provide pressure-controlled ventilation and V-V hemofiltration. Accordingly, the trial entry criteria to be applied to patients presenting with severe ARDS also served as unified patient transfer criteria for utilization by referral hospitals (i.e., hospitals other than Glenfield Hospital and CTCs). Thus, the design of this controlled trial was pragmatic; it mirrored usual clinical practice (including the workflow of patient transfer) in the United Kingdom as far as possible.

The results of the CESAR trial, published in 2009, demonstrated a significantly higher rate of 6-month survival without severe disability in patients undergoing ECMO, as well as the cost-effectiveness of this treatment compared with conventional ventilation management.

This was the world's first RCT to support strongly the clinical usefulness of ECMO in adults with acute respiratory failure, and it convinced many clinicians previously skeptical of this life-support technique of its clinical usefulness. Of course, the successful therapeutic results with ECMO obtained in the United Kingdom may not be reproducible in other countries. Even in the United Kingdom, it is still unclear whether hospitals other than Glenfield Hospital could obtain comparable results. ECMO centers with as much experience as Glenfield Hospital may not exist everywhere in the world. ${ }^{44}$ The transport system for critically ill patients may also vary from country to country. These factors clearly affect the results obtained with ECMO. Despite these problems, however, clear establishment of previously unavailable evidence for the usefulness of ECMO in adults with acute respira- 
tory failure (an advanced life-support technique in the treatment of acute, potentially fatal illness for which it is extremely difficult to obtain clinical evidence in the setting of a rigorous clinical trial) even at only one particular hospital within a limited geographic area is an encouraging message to health care professionals engaged in the treatment of this life-threatening condition.

\section{Changes in survival rate achieved with ECMO}

A survival rate of $63 \%$ was achieved in adults receiving ECMO in the CESAR trial, and a comparable survival rate of approximately $60 \%(55 \%-76 \%)$ was also reported in earlier case series, summarized in Table 5. Since publication of the earliest of these case series in $1997,{ }^{41}$ advancement in various fields of science and technology have resulted in steady improvement of the components of ECMO systems, such as membrane oxygenators ${ }^{45}$ and centrifugal pumps. ${ }^{46}$ The number of clinical cases may have increased with time as well, with concomitant accumulation of treatment experience. Although these factors may well have improved the survival rate achieved by ECMO with time, the observed survival rate remains practically unchanged. This lack of improvement in the survival rate may be ascribable to changes in entry criteria for ECMO in clinical algorithms. ${ }^{13}$ It can be anticipated that changes in exclusion criteria (e.g., age, duration of mechanical ventilation prior to ECMO) to allow application of ECMO to a wider range of patients will make it more difficult to determine on initiation of ECMO whether respiratory failure in a particular patient is reversible, thereby increasing the number of patients refractory to ECMO therapy. Consistent with this, Brogan et al., who retrospectively reviewed the ELSO registry from 1986 to 2006 and analyzed pre-ECMO and ECMO parameters over time with the period of data acquisition divided into quartiles ${ }^{47}$ found that since 1992 the survival rate remained almost constant over time although hours of ventilation rapidly decreased, suggesting a trend toward earlier initiation of ECMO. In addition, the median age of patients gradually increased while the rates of pre-ECMO cardiac arrest, documented infections, and use of inotropic medication rapidly increased. ${ }^{47}$ These changes show that the indications for ECMO have been changing over time.

\section{Complications of ECMO}

The most problematic complication during ECMO is hemorrhage. As a part of an extensive analysis of the ELSO registry data, Brogan et al. examined complications associ- ated with ECMO in detail ${ }^{47}$ and reported an incidence of $42 \%$ for hemorrhage over the entire 20 -year period. An incidence of $54 \%$ was also reported for hemorrhagic complications during ECMO in patients with ARDS due to 2009 influenza A (H1N1) in Australia and New Zealand. ${ }^{6,48}$ The observed hemorrhagic complications include fatal ones such as intracranial hemorrhage. To minimize the risk of hemorrhagic complications and prevent thrombotic occlusion of the blood circuit, strict control of heparin dosing is required during ECMO. While the ELSO General Guidelines state that the activated clotting time (ACT) should be adjusted to 1.5 times normal for the ACT measurement system, Oliver ${ }^{49}$ pointed out the poor correlation between ACT and blood heparin levels and proposed the use of activated partial thromboplastin time (APTT) based on its better sensitivity to changes in blood heparin level. He stated that most agree that the APTT should be 1.5-2.5 times the control. ${ }^{49}$ Although ACT is routinely used in our ICU for control of anticoagulant level in extracorporeal circulation management, we often experience discrepancies between ACT measurements and actual degree of blood coagulation within the extracorporeal circuit. Because such a discrepancy may lead to hemorrhagic complications directly affecting clinical outcome, further investigation is needed to identify an optimal indicator for monitoring anticoagulation with heparin during ECMO and determine its range of values, ensuring successful anticoagulation management.

Another important category of complications associated with ECMO is infection. Hsu et al. recently reported a single-center 6-year study in which the incidence of nosocomial infection in patients receiving ECMO was similar to that in other ICU patients not receiving ECMO.$^{50}$ Notably, despite the advancement of ECMO devices over time, stimulated by advances in science and technology, analysis of the ELSO registry data by Brogan et al. ${ }^{47}$ failed to demonstrate a decrease in mechanical complications during the last quartile (2002-2006) compared with the earlier three quartiles. This suggests that the technological advancements achieved in the field of ECMO may not yet be sufficient to reduce mechanical complications. Although further improvement of ECMO devices to make them more user-friendly, easier, and safer to operate is desirable, efforts by all members of ECMO teams to improve the technical expertise and safety awareness is concomitantly required to minimize the risk of complications associated with ECMO.

\section{Pumpless gas exchange}

Interventional lung assist is a recently developed ECLS technique that uses a simple pumpless system and is 
expected to be easier and safer to perform than conventional ECMO. The membrane oxygenator, Novalung, which was newly developed for this technique, is based on heparin-coated hollow fiber technology with optimized blood flow by reducing resistance. It does not require a pump for operation because its connection to the patient via arterial and venous cannulas generates sufficient passive blood flow across the hollow fiber membrane driven by the patient's arteriovenous pressure gradient. Furthermore, the system runs at a lower dose of heparin anticoagulant owing to its improved biocompatibility. ${ }^{51}$ It is mainly intended for $\mathrm{CO}_{2}$ elimination but is capable of oxygenation to a certain extent.

While conventional pump-driven ECMO systems have been used exclusively in patients in life-threatening condition, this simple pumpless ILA system has the future potential for aggressive application to a wider variety of conditions as a safer respiratory support system provided it operates without a pump at a reduced anticoagulant dose and thereby reduces the risk of various complications including hemorrhage. Immediate initiation of ILA upon manifestation of hypercapnia at an early stage of ARDS and subsequent switching to ECMO upon advanced impairment of lung oxygenation could reduce VILI to an extent greater than ever and thereby improve the survival rate. ILA has achieved excellent clinical results mainly in Europe, and clinical trials of ILA are currently in progress in Japan. We hope for early completion and publication of a Japanese RCT of ILA as a useful option of respiratory support applicable to pre-ECMO treatment.

\section{Conclusion}

Stimulated by the success of the CESAR trial, ECMO for the treatment of respiratory failure can be expected to be performed in a greater number of medical institutions. In consequence, it will be necessary for hospitals not capable of performing ECMO to decide whether and when to transfer patients to other hospitals functioning as ECMO centers in accordance with the specific local health care environment. In turn, ECMO centers will have to acquire proficiency in appropriate management of all aspects of treatment including minimization of hemorrhage and mechanical complications, which will require organization of a multidisciplinary team. In addition, when ILA becomes available in clinical practice in Japan, a stepwise treatment algorithm for respiratory failure involving pumpless ILA and pump-driven conventional ECMO in series will be developed: respiratory support in patients with respiratory failure will be started with ILA and then switched to ECMO in response to changes in clinical condition. In any case, ECMO can be expected to gain importance as a technique for respiratory support.

\section{References}

1. Bernard GR, Artigas A, Brigham KL, Carlet J, Falke K, Hudson L, et al. The American-European Consensus Conference on ARDS: definitions, mechanisms, relevant outcomes, and clinical trial coordination. Am J Respir Crit Care Med 1994; 149:818-24.

2. Hemmila MR, Napolitano LM. Severe respiratory failure: advanced treatment options. Crit Care Med 2006;34:27890.

3. Peek GJ, Mugford M, Tiruvoipati R, Wilson A, Allen E, Thalanany MM, et al. Efficacy and economic assessment of conventional ventilatory support versus extracorporeal membrane oxygenation for severe adult respiratory failure (CESAR): a multicentre randomised controlled trial. Lancet 2009;374:1351-63.

4. Freed DH, Henzler D, White CW, Fowler R, Zarychanski R, Hutchison $\mathrm{J}$, et al. Extracorporeal lung support for patients who had severe respiratory failure secondary to influenza A (H1N1) 2009 infection in Canada. Can J Anaesth 2010; 57:240-7.

5. Roch A, Lepaul-Ercole R, Grisoli D, Bessereau J, Brissy O, Castanier M, et al. Extracorporeal membrane oxygenation for severe influenza A (H1N1) acute respiratory distress syndrome: a prospective observational comparative study. Intensive Care Med 2010;36:1899-905.

6. Australia and New Zealand Extracorporeal Membrane Oxygenation Influenza Investigators. Extracorporeal membrane oxygenation for 2009 influenza A (H1N1) acute respiratory distress syndrome. JAMA 2009;302:E1-8.

7. Chou NK, Chen YS, Ko WJ, Huang SC, Chao A, Jan GJ, et al. Application of extracorporeal membrane oxygenation in adult burn patients. Artif Organs 2001;25:622-6.

8. Cordell-Smith JA, Roberts N, Peek GJ, Firmin RK. Traumatic lung injury treated by extracorporeal membrane oxygenation (ECMO). Injury 2006;37:29-32.

9. Hämmäinen $P$, Schersten $H$, Lemström $K$, Riise GC, Kukkonen S, Swärd K, et al. Usefulness of extracorporeal membrane oxygenation as a bridge to lung transplantation: a descriptive study. J Heart Lung Transplant 2011;30: 103-7.

10. Mason DP, Boffa DJ, Murthy SC, Gildea TR, Budev MM, Mehta AC, et al. Extended use of extracorporeal membrane oxygenation after lung transplantation. J Thorac Cardiovasc Surg 2006;132:954-60.

11. Nishinaka T, Tatsumi E, Katagiri N, Ohnishi H, Mizuno T, Shioya K, et al. Up to 151 days of continuous animal perfusion with trivial heparin infusion by the application of a longterm durable antithrombogenic coating to a combination of a seal-less centrifugal pump and a diffusion membrane oxygenator. J Artif Organs 2007;10:240-4.

12. Bermudez CA, Rocha RV, Sappington PL, Toyoda Y, Murray $\mathrm{HN}$, Boujoukos AJ. Initial experience with single cannulation for venovenous extracorporeal oxygenation in adults. Ann Thorac Surg 2010;90:991-5.

13. Hemmila MR, Rowe SA, Boules TN, Miskulin J, McGillicuddy JW, Schuerer DJ, et al. Extracorporeal life support for severe acute respiratory distress syndrome in adults. Ann Surg 2004:240:595-607. 
14. Peek GJ. ECLS for adult respiratory failure: etiology and indications. In: Van Meurs K, Lally KP, PeeK G, Zwischenberger JB, editors. ECMO extracorporeal cardiopulmonary support in critical care. 3rd edn. Ann Arbor: Extracorporeal Life Support Organization; 2005. p. 393-402.

15. Rich PB, Awad SS, Crotti S, Hirschl RB, Bartlett RH, Schreiner RJ. A prospective comparison of atrio-femoral and femoro-atrial flow in adult venovenous extracorporeal life support. J Thorac Cardiovasc Surg 1998;116:62832.

16. Curtis JJ, Walls JT, Wagner-Mann CC, Schmaltz RA, Demmy TL, McKenney CA, et al. Centrifugal pumps: description of devices and surgical techniques. Ann Thorac Surg 1999;68: $666-71$

17. Lawson DS, Ing R, Cheifetz IM, Walczak R, Craig D, Schulman $\mathrm{S}$, et al. Hemolytic characteristics of three commercially available centrifugal blood pumps. Pediatr Crit Care Med 2005;6:573-7.

18. Kawahito K, Nosé Y. Hemolysis in different centrifugal pumps. Artif Organs 1997;21:323-6.

19. Rawn DJ, Harris HK, Riley JB, Yoda DN, Blackwell MM. An under-occluded roller pump is less hemolytic than a centrifugal pump. J Extra Corpor Technol 1997;29: $15-8$.

20. Tatsumi E. Artificial lungs: current state and trends of clinical use and research and development. J Artif Organs 2007; 10:1-5.

21. Hill JD, O’Brien TG, Murray JJ, Dontigny L, Bramson ML, Osborn JJ, et al. Prolonged extracorporeal oxygenation for acute post-traumatic respiratory failure (shock-lung syndrome): use of the Bramson membrane lung. N Engl J Med 1972;286:629-34.

22. Zapol WM, Snider MT, Hill JD, Fallat RJ, Bartlett RH, Edmunds LH, et al. Extracorporeal membrane oxygenation in severe acute respiratory failure: a randomized prospective study. JAMA 1979;242:2193-6.

23. Schuerer DJ, Kolovos NS, Boyd KV, Coopersmith CM. Extracorporeal membrane oxygenation: current clinical practice, coding, and reimbursement. Chest 2008;134:179 84.

24. Bartlett RH, Roloff DW, Cornell RG, Andrews AF, Dillon PW, Zwischenberger JB. Extracorporeal circulation in neonatal respiratory failure: a prospective randomized study. Pediatrics 1985;76:479-87.

25. Swaniker F, Kolla S, Moler F, Custer J, Grams R, Barlett R, et al. Extracorporeal life support outcome for 128 pediatric patients with respiratory failure. J Pediatr Surg 2000;35: 197-202.

26. Adolph V, Heaton J, Steiner R, Bonis S, Falterman K, Arensman R. Extracorporeal membrane oxygenation for nonneonatal respiratory failure. J Pediatr Surg 1991;26: 326-32.

27. Lillehei CW, O'Rourke PP, Vacanti JP, Crone RK. Role of extracorporeal membrane oxygenation in selected pediatric respiratory problems. J Thorac Cardiovasc Surg 1989;98: 968-70.

28. UK Collaborative ECMO Trail Group. UK collaborative randomised trial of neonatal extracorporeal membrane oxygenation. Lancet 1996;348:75-82.

29. Acute Respiratory Distress Syndrome Network. Ventilation with lower tidal volumes as compared with traditional tidal volumes for acute lung injury and the acute respiratory distress syndrome. N Engl J Med 2000;342:1301-8.

30. Tremblay LN, Slutsky AS. Ventilator-induced lung injury: from the bench to the bedside. Intensive Care Med 2006;32: 24-33.
31. Stawicki SP, Goyal M, Sarani B. High-frequency oscillatory ventilation (HFOV) and airway pressure release ventilation (APRV): a practical guide. J Intensive Care Med 2009;24: 215-29.

32. Steinberg KP, Hudson LD, Goodman RB, Hough CL, Lanken PN, Hyzy R, et al. Efficacy and safety of corticosteroids for persistent acute respiratory distress syndrome. $\mathrm{N}$ Engl J Med 2006;354:1671-84.

33. Gattinoni L, Carlesso E, Taccone P, Polli F, Guérin C, Mancebo J. Prone positioning improves survival in severe ARDS: a pathophysiologic review and individual patient meta-analysis. Minerva Anestesiol 2010;76:448-54.

34. Taylor RW, Zimmerman JL, Dellinger RP, Straube RC, Criner GJ, Davis K Jr, et al. Low-dose inhaled nitric oxide in patients with acute lung injury: a randomized controlled trial. JAMA 2004;291:1603-9.

35. Lewandowski K, Rossaint R, Pappert D, Gerlach H, Slama $\mathrm{KJ}$, Weidemann $\mathrm{H}$, et al. High survival rate in 122 ARDS patients managed according to a clinical algorithm including extracorporeal membrane oxygenation. Intensive Care Med 1997;23:819-35.

36. Bartlett RH, Morris AH, Fairley HB, Hirsch R, O'Connor N, Pontoppidan $\mathrm{H}$. A prospective study of acute hypoxic respiratory failure. Chest 1986;89;684-9.

37. Vasilyev S, Schaap RN, Mortensen JD. Hospital survival rates of patients with acute respiratory failure in modern respiratory intensive care units: an international, multicenter, prospective survey. Chest 1995;107:1083-8.

38. Ullrich R, Lorber C, Röder G, Urak G, Faryniak B, Sladen RN, et al. Controlled airway pressure therapy, nitric oxide inhalation, prone position, and extracorporeal membrane oxygenation (ECMO) as components of an integrated approach to ARDS. Anesthesiology 1999;91: $1577-86$.

39. Mols G, Loop T, Geiger K, Farthmann E, Benzing A. Extracorporeal membrane oxygenation: a ten-year experience. Am J Surg 2000;180:144-54.

40. Beiderlinden M, Eikermann M, Boes T, Breitfeld C, Peters J. Treatment of severe acute respiratory distress syndrome: role of extracorporeal gas exchange. Intensive Care Med 2006;32: 1627-31.

41. Peek GJ, Moore HM, Moore N, Sosnowski AW, Firmin RK. Extracorporeal membrane oxygenation for adult respiratory failure. Chest 1997;112:759-64.

42. Lindén V, Palmér K, Reinhard J, Westman R, Ehrén H, Granholm $\mathrm{T}$, et al. High survival in adult patients with acute respiratory distress syndrome treated by extracorporeal membrane oxygenation, minimal sedation, and pressure supported ventilation. Intensive Care Med 2000;26:1630-7.

43. Nehra D, Goldstein AM, Doody DP, Ryan DP, Chang Y, Masiakos PT. Extracorporeal membrane oxygenation for nonneonatal acute respiratory failure: the Massachusetts General Hospital experience from 1990 to 2008. Arch Surg 2009;144:427-32.

44. Betit P. Extracorporeal membrane oxygenation: quo vadis? Respir Care 2009;54:948-57.

45. Iwahashi H, Yuri K, Nose Y. Development of the oxygenator: past, present, and future. J Artif Organs 2004; 7:111-20.

46. Horton S, Thuys C, Bennett M, Augustin S, Rosenberg M, Brizard C. Experience with the Jostra Rotaflow and QuadroxD oxygenator for ECMO. Perfusion 2004;19:17-23.

47. Brogan TV, Thiagarajan RR, Rycus PT, Bartlett RH, Bartton SL. Extracorporeal membrane oxygenation in adults with severe respiratory failure: a multi-center database. Intensive Care Med 2009;35:2105-14. 
48. Wong I, Vuylsteke A. Use of extracorporeal life support to support patients with acute respiratory distress syndrome due to N1H1/2009 influenza and other respiratory infections. Perfusion 2011;26:7-20.

49. Oliver WC. Anticoagulation and coagulation management for ECMO. Semin Cardiothorac Vasc Anesth 2009;13: $154-75$.
50. Hsu MS, Chiu KM, Huang YT, Kao KL, Chu SH, Liao CH. Risk factors for nosocomial infection during extracorporeal membrane oxygenation. J Hosp Infect 2009;73:210-6.

51. Bein T, Weber F, Philipp A, Prasser C, Pfeifer M, Schmid FX, et al. A new pumpless extracorporeal interventional lung assist in critical hypoxemia/hypercapnia. Crit Care Med 2006;34: $1372-7$. 